\title{
DEPENDENCE OF EXCITON BINDING ENERGY ON MAGNETIC FIELD IN PARABOLIC QUANTUM WELL MADE OF DILUTED MAGNETIC SEMICONDUCTORS
}

\author{
F. KYRYCHENKo AND J. KossuT \\ Institute of Physics, Polish Academy of Sciences \\ Al. Lotników 32/46, 02-668 Warsaw, Poland
}

\begin{abstract}
Influence of an external magnetic field on exciton binding in parabolic quantum wells composed of diluted magnetic semiconductors are studied variationally using separable trial wave functions. The effect is due to a change of shape of the confining potential induced by an external magnetic field. The validity of the results of the calculations is checked by solving numerically the corresponding one-dimensional Schrödinger equations. Further, we check the results by performing variational calculations in the case of the Ex11 exciton (i.e. with electron and hole in their ground subbands) with a non-separable trial wave function. It is shown that, contrary to the case of a rectangular quantum well, for the parabolic quantum well the magnetic field dependence of the binding energy is significant.
\end{abstract}

PACS numbers: $71.35 . \mathrm{Cc}, 73.20 . \mathrm{Dx}$

Excitons in semiconductor parabolic quantum wells (PQW) have been studied theoretically earlier by variational methods [1]. The aim of this work is to include into considerations the presence of an external magnetic field in the case of diluted magnetic semiconductor PQW which can change a shape of the confining potential. Such structures have been recently grown and investigated experimentally [2]. Our present calculations will assume material parameters corresponding to actual $\mathrm{CdTe} / \mathrm{Cd}_{0.2} \mathrm{Mn}_{0.8} \mathrm{Te} \mathrm{PQW}$ structures, namely: electron effective mass $m_{\mathrm{e}}^{*}=0.09 m_{0}$, heavy hole effective masses $m_{\mathrm{hh}}^{z}=0.65 m_{0}$ in the growth direction, and $m_{\mathrm{hh}}^{x-y}=0.15 m_{0}$ in-plane mass, valence band offset parameter $Q=0.3$ and the dielectric constant $\varepsilon=9.7$.

We neglect the direct influence of the magnetic field on the electron and hole kinetic energy, leaving only the magnetic field induced shift of the band edges related to a strong exchange interaction characteristic of diluted magnetic semiconductors (DMS). Then, the Hamiltonian can be written in the following usual form: 


$$
\begin{aligned}
\widehat{H}_{\mathrm{ex}} & =\widehat{H}_{\mathrm{e}}\left(z_{\mathrm{e}}\right)+\widehat{H}_{\mathrm{h}}\left(z_{\mathrm{h}}\right)-\frac{\hbar}{2 \mu}\left[\frac{1}{\rho} \frac{\partial}{\partial \rho} \rho \frac{\partial}{\partial \rho}+\frac{1}{\rho^{2}} \frac{\partial^{2}}{\partial \varphi^{2}}\right] \\
& -\frac{e^{2}}{4 \pi \varepsilon \varepsilon_{0} \sqrt{\rho^{2}+\left(z_{\mathrm{e}}-z_{\mathrm{h}}\right)^{2}}}
\end{aligned}
$$

where $\mu$ is exciton reduced mass in $x-y$ plane, while $\rho=\sqrt{\left(x_{\mathrm{e}}-x_{\mathrm{h}}\right)^{2}+\left(y_{\mathrm{e}}-y_{\mathrm{h}}\right)^{2}}$ is the electron-hole distance in the $x-y$ plane. The first two terms in Eq. (1) describe motions in the $z$-direction (i.e., along the growth axis) of an electron and a hole, respectively, non-interacting with each other. As mentioned, we assume the magnetic field does influence only the potential shape in the $z$-direction. The trial wave function can be taken in the form

$$
\Psi_{n, m}^{\mathrm{ex} 2 \mathrm{D}}\left(z_{\mathrm{e}}, z_{\mathrm{h}}, \rho\right)=\sqrt{\frac{2 \lambda^{2}}{\pi}} \Psi_{n}^{\mathrm{e}}\left(z_{\mathrm{e}}\right) \Psi_{m}^{\mathrm{h}}\left(z_{\mathrm{h}}\right) \exp (-\lambda \rho) .
$$

The function contains the exponential term which corresponds to $2 \mathrm{D}$ electron-hole relative motion in $x-y$ plane with the variational parameter $\lambda ; \Psi_{n}^{\mathrm{e}}\left(z_{\mathrm{e}}\right), \Psi_{m}^{\mathrm{h}}\left(z_{\mathrm{h}}\right)$ are $n$-th and $m$-th eigenfunctions of $\widehat{H}_{\mathrm{e}}\left(z_{\mathrm{e}}\right)$ and $\widehat{H}_{\mathrm{h}}\left(z_{\mathrm{h}}\right)$, respectively.

We define the exciton binding energy as a difference between the calculated total exciton energy, as resulting from the minimization of the Hamiltonian matrix element and the sum of the electron and the hole confinement energies without the Coulomb interaction

$$
E_{\text {bind }}=\min \left\langle\Psi^{\mathrm{ex}}\left|\widehat{H}_{\mathrm{ex}}\right| \Psi^{\mathrm{ex}}\right\rangle-E_{\mathrm{e}}-E_{\mathrm{h}} .
$$

In the case of the PQW made of DMS in a magnetic field we face the problem of determination of the one-dimensional wave functions $\Psi_{n}^{\mathrm{e}}\left(z_{\mathrm{e}}\right), \Psi_{m}^{\mathrm{h}}\left(z_{\mathrm{h}}\right)$. As mentioned, the potential shape for each type of the carriers becomes non-parabolic and a simple 1D harmonic oscillator wave functions, which we use in the case of a vanishing magnetic field, are not eigenfunctions of the problem anymore. In our calculations in the presence of a magnetic field we assume the wave functions to have an oscillator-like form but with parameters in the exponents to be obtained variationally. Therefore, $n$-th and $m$-th oscillator-like trial wave functions for the one-dimensional Schrödinger equations for the electron and the hole have the following form:

$$
\Psi_{\mathrm{e}}^{n}=A_{n} H_{n}\left(z_{\mathrm{e}}\right) \exp \left(-\alpha_{n} z_{\mathrm{e}}^{2}\right), \quad \Psi_{\mathrm{h}}^{m}=A_{m} H_{m}\left(z_{\mathrm{h}}\right) \exp \left(-\beta_{m} z_{\mathrm{h}}^{2}\right),
$$

where $\alpha_{n}, \beta_{m}$ are variational parameters, $H_{n}(z)$ are polynomials which allow us to preserve the orthogonality of the wave functions with different values of $n$ and $m$. In the case of infinitely deep PQW when $\alpha_{n}$ (or $\beta_{m}$ ) are constant for all electronic (hole) eigenstates these are Hermite polynomials. When $\alpha_{n}$ (or $\beta_{m}$ ) depends on $n$ (or $m$ ), the polynomials have to be calculated directly to assume the orthogonality. To check the results of the variational calculations we compared them with those obtained by numerical solutions of one-dimensional Schrödinger equations obtained by "shooting method".

In Fig. 1 we plot the results of this comparison for the first three heavy hole spin up confined states in $82 \mathrm{ML}$ wide PQW in the magnetic field $H=5 \mathrm{~T}$ applied along the growth direction (for the heavy hole spin up subsystem, the influence 


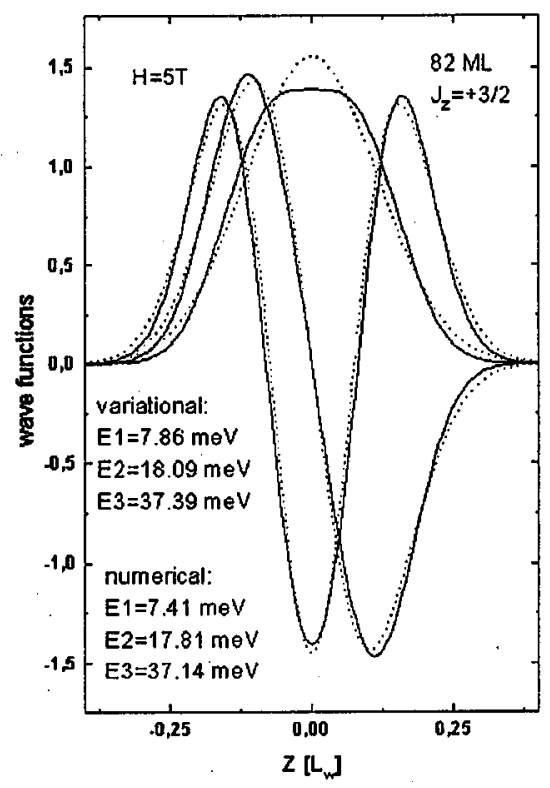

Fig. 1. Eigenenergies and wave functions for ground, first and second excited heavy hole spin up states in $82 \mathrm{ML}$ wide $\mathrm{PQW}$ in the magnetic field $H=5 \mathrm{~T}$. Solid curves were obtained by numerical solution of the Schrödinger equation, dotted lines correspond to wave functions calculated variationally.

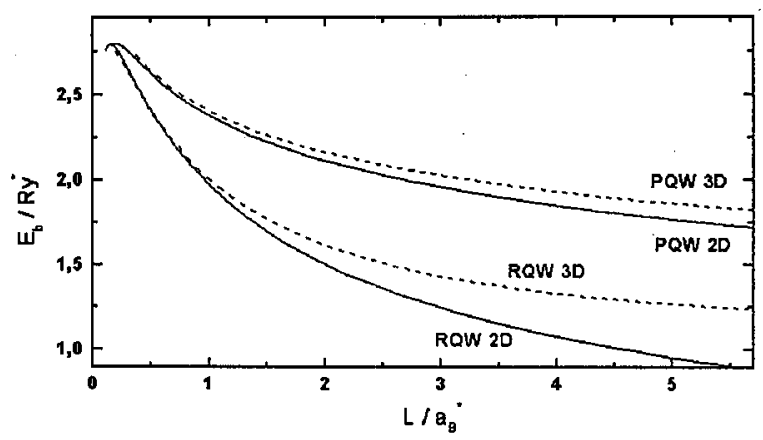

Fig. 2. Exciton binding energies (in units of effective 3D excitonic Rydberg) plotted vs. well width (in units of 3D excitonic Bohr radius) for parabolic and rectangular quantum wells. The solid curves were obtained using separable trial wave functions while the dashed curves represent results obtained with non-separable wave functions.

of the magnetic field on the potential shape is the most significant). We can see that the energies of the first and second excited states and their wave functions obtained variationally and numerically are very close to each other. A slightly greater difference occurs in the case of the ground state. 


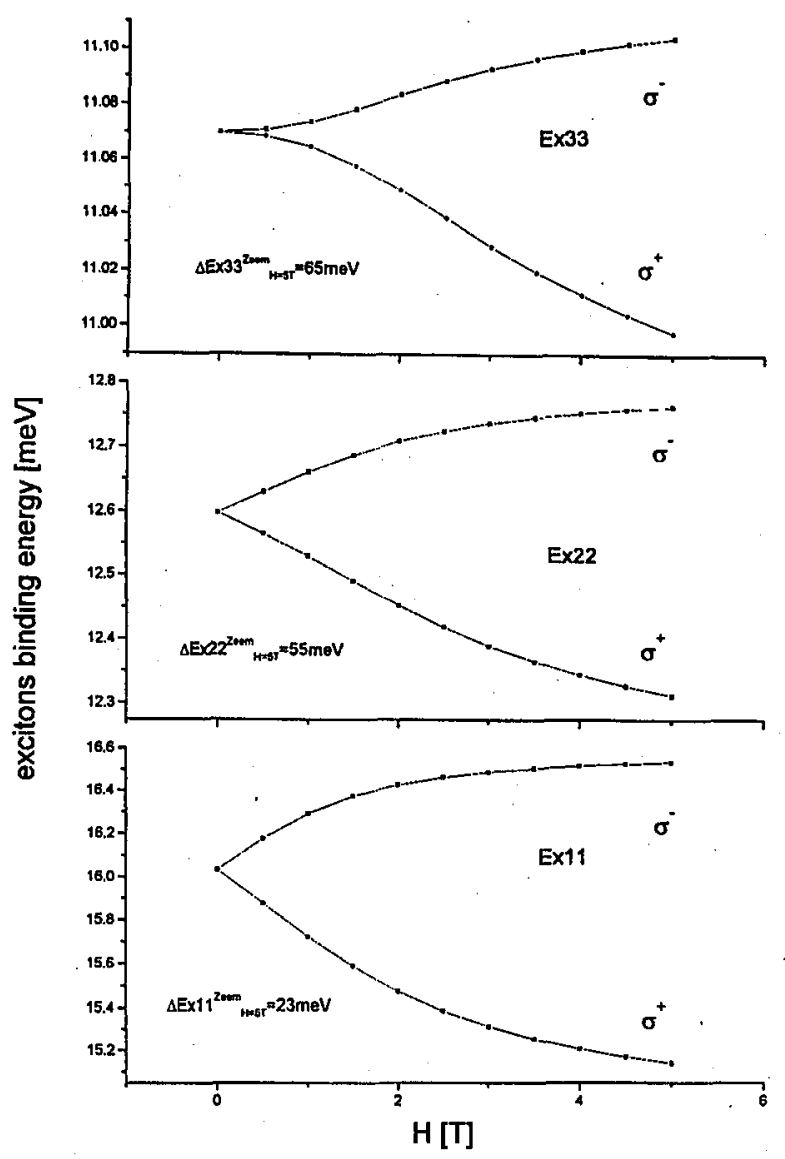

Fig. 3. Magnetic field dependence of the exciton binding energy for $82 \mathrm{ML}$ wide PQW for Ex11, Ex22, Ex33 excitons (i.e., excitons comprising electron and hole on their ground, first and second excited subbands, respectively). The labels $\sigma^{+}, \sigma^{-}$correspond to excitons, excited by different light polarizations. The value of the corresponding total exciton Zeeman splitting $\Delta E x_{N N}^{\text {Zeem }}$ of the exciton state in valving the hole and the electron in $N$-th confined state in the magnetic field $H=5 \mathrm{~T}$ is given in each panel.

The next problem is connected to the fact that Eq. (2) separates completely the 2D electron-hole relative motion from the motion in the $z$-direction. As it was shown in Ref. [3] such choice of the trial wave function leads to incorrect results for wide (wider than effective $3 \mathrm{D}$ Bohr radius) rectangular quantum wells. At the same time the actual PQW structures are relatively wide because of the method of their fabrication [2]. To check our results we perform variational calculations of the exciton binding energy for Ex11 (i.e., with the electron and hole associated with their ground subbands) using the non-separable trial wave function

$$
\Psi^{\operatorname{ex} 3 \mathrm{D}}\left(z_{\mathrm{e}}, z_{\mathrm{h}}, \rho\right)=N \exp \left(-\alpha z_{\mathrm{e}}^{2}\right) \exp \left(-\beta z_{\mathrm{h}}^{2}\right) \exp \left(-\lambda \sqrt{\rho^{2}+\left(z_{\mathrm{e}}-z_{\mathrm{h}}\right)^{2}}\right) .
$$


Here $N$ is the normalization constant and the minimization is performed by varying three parameters $\alpha, \beta, \lambda$ simultaneously. The wave function (4) contains the term corresponding to 3D electron-hole relative motion and, as shown in Ref. [3] in the case of wide rectangular quantum wells, is expected to give more accurate results. Figure 2 shows the plot of the exciton binding energy for the electron and the hole in their ground subbands calculated for two above-mentioned types of the trial wave functions. The solid line shows the results for the separable 2D-like wave function (2) and the dashed line represents those for the non-separable 3D-like function (4). The units in Fig. 2 are 3D exciton rydberg $R y^{*}$ and Bohr radius $a_{\mathrm{B}}^{*}$ (in our case $R y^{*}=8.11 \mathrm{meV}, a_{\mathrm{B}}^{*}=91.5 \AA$ ). For comparison we plotted also the exciton binding energy calculated variationally for rectangular quantum wells with the same parameters using a separable (solid line) and non-separable (dashed line) trial wave functions. We can see that the confinement is effectively stronger in the case of PQW resulting in the binding energy that is greater than in the case of rectangular quantum wells (RQW) with the same width, thus, leading to a smaller difference between the results of the calculations using separable and non-separable form of the trial wave functions. It is important to note that the same calculations performed in the presence of a magnetic field showed that the difference between results of the calculation using separable and non-separable trial wave functions does not increase with the magnetic field up to $5 \mathrm{~T}$.

In Fig. 3 we plot the results of the calculation of the exciton binding energy as a function of magnetic field applied along the growth direction for 82 monolayer wide $\mathrm{CdTe} / \mathrm{Cd}_{0.2} \mathrm{Mn}_{0.8} \mathrm{Te} \mathrm{PQW}$ for Ex11, Ex22, Ex33 (i.e., excitons associated with electrons and holes on their ground, first and second excited confined subbands) excited by light different circular polarizations. We can see that, contrary to the case of very deep rectangular quantum wells when the exciton binding energy hardly depends on magnetic field (again assuming that the magnetic field does not affect the kinetic energy), for DMS parabolic quantum wells this dependence is more significant. The variation is most pronounced for heavy hole excitons in their ground state (Ex11) in which case the difference between the excitons excited by $\sigma^{-}$and $\sigma^{+}$polarizations reaches $1.5 \mathrm{meV}$ in the magnetic field $H=5 \mathrm{~T}$.

This work is supported in part by the Committee for Scientific Research (Poland) through grant 8T11B 01411 and partly by the Volkswagen Stiftung.

\section{References}

[1] Yang Chu-liang, Yang Qing, Phys. Rev, B 37, 1364 (1988).

[2] T. Wojtowicz, M. Kutrowski, G. Cywiński, E. Dynowska, G. Karczewski, J. Kossut, R. Fiederling, G. Mackh, U. Zehnder, W. Ossau, in: Proc. XXV Int. School on Physics of Semiconducting Compounds (Jaszowiec 1996), Acta Phys. Pol. A 90, 977 (1996).

[3] G. Bastard, E.E. Mendez, L.L. Chang, L. Esaki, Phys. Rev. B 26, 1974 (1982). 